\title{
The SIR2 gene family, conserved from bacteria to humans, functions in silencing, cell cycle progression, and chromosome stability
}

\author{
Carrie Baker Brachmann, Joyce M. Sherman, ${ }^{1}$ Scott E. Devine, Elizabeth E. Cameron, \\ Lorraine Pillus, ${ }^{1}$ and Jef D. Boeke \\ Department of Molecular Biology and Genetics, Johns Hopkins School of Medicine, Baltimore, Maryland 21205; \\ ${ }^{1}$ Department of Molecular, Cellular and Developmental Biology, Porter Biosciences, University of Colorado, CB347, Boulder, \\ Colorado 80309 USA
}

\begin{abstract}
Genomic silencing is a fundamental mechanism of transcriptional regulation, yet little is known about conserved mechanisms of silencing. We report here the discovery of four Saccharomyces cerevisiae homologs of the SIR2 silencing gene (HSTs), as well as conservation of this gene family from bacteria to mammals. At least three $H S T$ genes can function in silencing; HST1 overexpression restores transcriptional silencing to a sir2 mutant and hst 3 hst 4 double mutants are defective in telomeric silencing. In addition, HST3 and HST4 together contribute to proper cell cycle progression, radiation resistance, and genomic stability, establishing new connections between silencing and these fundamental cellular processes.
\end{abstract}

[Key Words: HST genes; Saccharomyces cerevisiae; telomeric silencing; homologous recombination; chromosome segregation]

Received September 13, 1995; revised version accepted October 13, 1995.

Transcriptional inactivation of large genomic regions, or silencing, is an important and highly conserved mechanism of gene regulation. This regional, gene nonspecific form of repression is often associated with local alterations in chromatin structure. For example, heterochromatin is associated with transcriptional repression and has been well-documented at the Drosophila chromocenter and telomeres (Weiler and Wakimoto 1995), at mouse (Butner and Lo 1986) and Schizosaccharomyces pombe (Allshire et al. 1994; Nimmo et al. 1994) centromeres and telomeres, and on the mammalian inactive X chromosome (Migeon 1994). In Drosophila, breakpoints creating novel junctions between heterochromatic and euchromatic regions result in mosaic patterns of gene activity called position effect variegation (PEV). Finally, apparently Bacillus subtilis can exist with two chromosomes, only one of which is transcriptionally active (Schaeffer et al. 1976; Guillen et al. 1985).

In Saccharomyces, at least two types of genomic regions are silenced, telomeres and the silent $H M$ matingtype loci. In both cases, silencing occurs independently of the gene being silenced, and displays an epigenetic or variegating component (Schnell and Rine 1986; Mahoney and Broach 1989; Pillus and Rine 1989; Gottschling et al. 1990; Sussel and Shore 1991; Sussel et al. 1993). Silenced loci are inaccessible to DNA modifying enzymes both in vivo and in isolated nuclei
(Gottschling 1992; Singh and Klar 1992; Wright et al. 1992; Loo and Rine 1994), supporting the idea that silencing is associated with altered chromatin structure. Although cis-regulatory elements differ between the $H M$ loci and telomeres, essential trans-acting factors are shared (Laurenson and Rine 1992). In particular, Sir2, Sir3, and Sir4 proteins are necessary for silencing, and participate with Raplp and histones $\mathrm{H} 3$ and $\mathrm{H} 4$ in determining chromosomal structure and nuclear organization (for review, see Moretti et al. 1994; Shore 1994; Cockell et al. 1995; and Hecht et al. 1995). However, none of these interactions appear mediated through direct binding of Sir proteins to DNA (Buchman et al. 1988).

Recent data identifying mutations in subunits of the origin recognition complex (ORC; Bell et al. 1993) causing both silencing and cell cycle defects (Foss et al. 1993; Micklem et al. 1993; Loo and Rine 1994, 1995) point to direct mechanistic connections between DNA replication and silencing. Similarly, recent experiments suggest that delays in cell cycle progression can influence silencing (Laman et al. 1995) and that the $G_{2} / M$ transition may represent a particularly vulnerable time for factors to activate silenced chromatin (Aparicio and Gottschling 1994). Exactly how the SIR genes, ORC, cis-regulatory regions, and other factors work together to promote transcriptional silencing is not yet clear.

Like the other $S I R$ genes, SIR2 shares an essential 
function in silencing both at the HM loci and telomeres, yet in particular, SIR2 has several unique and distinguishing characteristics. SIR2 suppresses rDNA recombination (Gottlieb and Esposito 1989) and may participate in regulation of histone deacetylation (Braunstein et al. 1993).

We and others (Derbyshire et al. 1995) have discovered a gene family in Saccharomyces cerevisiae with homology ranging from $30 \%$ to $63 \%$ amino acid identity with SIR2. In our search for SIR2 homologs we have also identified potential homologs from a bacterium as well as from plants and mammals. None of the yeast HST genes (homologous to SIR2) result in a $\mathrm{Sir}^{-}$reduced mating phenotype when mutated. However, increasing HST1 gene dosage significantly restores silencing to a sir2 mutant, and a hst 3 hst 4 double mutant is defective in telomeric silencing. These results indicate that although HST genes are not fully redundant with SIR2, they can participate in silencing.

We have also determined that the hst 3 hst 4 double mutant has a number of defects in addition to those affecting telomeric silencing. In this strain, cells accumulate with $G_{2}$ DNA content at the $G_{2} / M$ boundary. The interaction of hst 3 hst 4 mutations with the checkpoint control mutation rad9 was examined and a dramatic synthetic UV sensitivity phenotype was observed. We find that the hst 3 hst 4 double mutant exhibits significantly elevated rates of mitotic recombination, and chromosome missegregation. These phenotypes indicate that in addition to telomeric silencing, Hst3p and Hst4p play important roles in the cell cycle, DNA damage control, recombination, and genomic maintenance. The discovery and characterization of the conserved SIR2 gene family suggests that silencing proteins, first identified in yeast, may have general functions in cell cycle progression and genomic integrity.

\section{Results}

Identification of a novel family of yeast HST genes homologous to SIR2

We have discovered four new members of a SIR2 gene family through a combination of strategies including low-stringency hybridization, degenerate PCR, and data base analysis. These Saccharomyces genes encode proteins closely related by amino acid sequence. We have determined by Northern blot analysis using gene-specific probes that all of the HST genes are expressed /data not shown). None of the HST genes are essential; S. cerevisiae with complete knockouts in any of these genes, whether singly or in combination (including the quintuple null sir2 hst 1 hst2 hst 3 hst 4 mutant), is viable. However, some synthetic phenotypes are observed with various null mutant combinations and are described below.

The SIR2 gene family also includes related sequences in a variety of eukaryotes (human, rat, mouse, Caenorhabditis elegans, Arabidopsis, and fission yeast) and the prokaryote Staphylococcus aureus. Figure 1A shows an alignment of the five $S$. cerevisiae protein sequences as well as homologs from the budding yeast Kluyvero- myces lactis (Chen and Clark-Walker 1994), S. aureus, Rattus norvegicus, and Homo sapiens (see Materials and methods for details of sequence discovery and isolation). There are several highly conserved domains within the family, in particular the region that we have defined as the core domain (bounded by arrowheads in Fig. 1A). Although the core domain is well-conserved among family members, the relative lengths of the terminal domains of these proteins is quite variable (Fig. 1B). Alignment of the yeast proteins with homologs from other species reveals three subfamilies. One consists of Sir2p, Hst $1 \mathrm{p}$, and $K$. lactis Sir $2 \mathrm{p}$, and has a long, partially conserved amino-terminal domain. The second contains Hst $2 p$ and the mammalian and C. elegans sequences and the third contains Hst3p and Hst4p.

\section{Conserved amino acid sequence features of Sir2 and the Hst proteins}

Two amino acid sequence motifs are diagnostic for the Sir2 protein family: GAGISTS(L/A)GIPDFR and YTQNID. These amino acid coding regions formed the basis for degenerate PCR primers (Fig, 1A) and should be useful for identifying additional family members, should any exist. There are a number of other amino acid motifs revealed by the multiple sequence alignment. A previous study (Chen and Clark-Walker 1994) noted that Sir2p and KlSir2p contain motifs typical of DNA-binding proteins. Specifically, they contain a cluster of cysteine residues with unique spacing suggestive of a zinc finger and two potential leucine zippers. The four cysteine residues of the proposed metal-binding motif are conserved in all of the HST sequences (Fig. 1A, asterisks) and suggest that these residues may be functionally important. Although at least one leucine zipper may also be present in $S$. aureus Sir2p (SaSir2p), Hst $2 p$, and Hst $3 p$, it is interesting to note that the two putative leucine zipper motifs are conserved only in the most Sir2p-like family membersnamely Sir $2 p$ itself, KlSir $2 p$, and Hstlp. Many DNAbinding proteins contain these motifs.

\section{HST1 overexpression restores silencing at HMR}

Single hst null mutants, as well as hst 3 hst 4 null mutants mate as well as wild type, in both MATa and MATa backgrounds, indicating normal $H M$ locus silencing in these strains. To determine whether the HSTs share functional similarity with SIR2, the ability of the Hst proteins when overexpressed to complement the mating defect in a sir2 null mutant background was tested. The MATa and MATa sir2 strains LPY11 and LPY1393, respectively (see Table 1 for strain descriptions), were transformed with $2 \mu$ plasmids carrying HST1, HST2, $H S T 3$, or SIR2. Equal numbers of cells from logarithmically growing cultures of the LPY11 and LPY1393 transformants were spotted onto lawns of the mating tester strains LPY143 and LPY142, respectively (MAT $\alpha$ and MATa). Overexpression of HST1 (Fig. 2), but not of HST2 or HST3 (data not shown), complemented the sir2 mu- 
Brachmann et al.

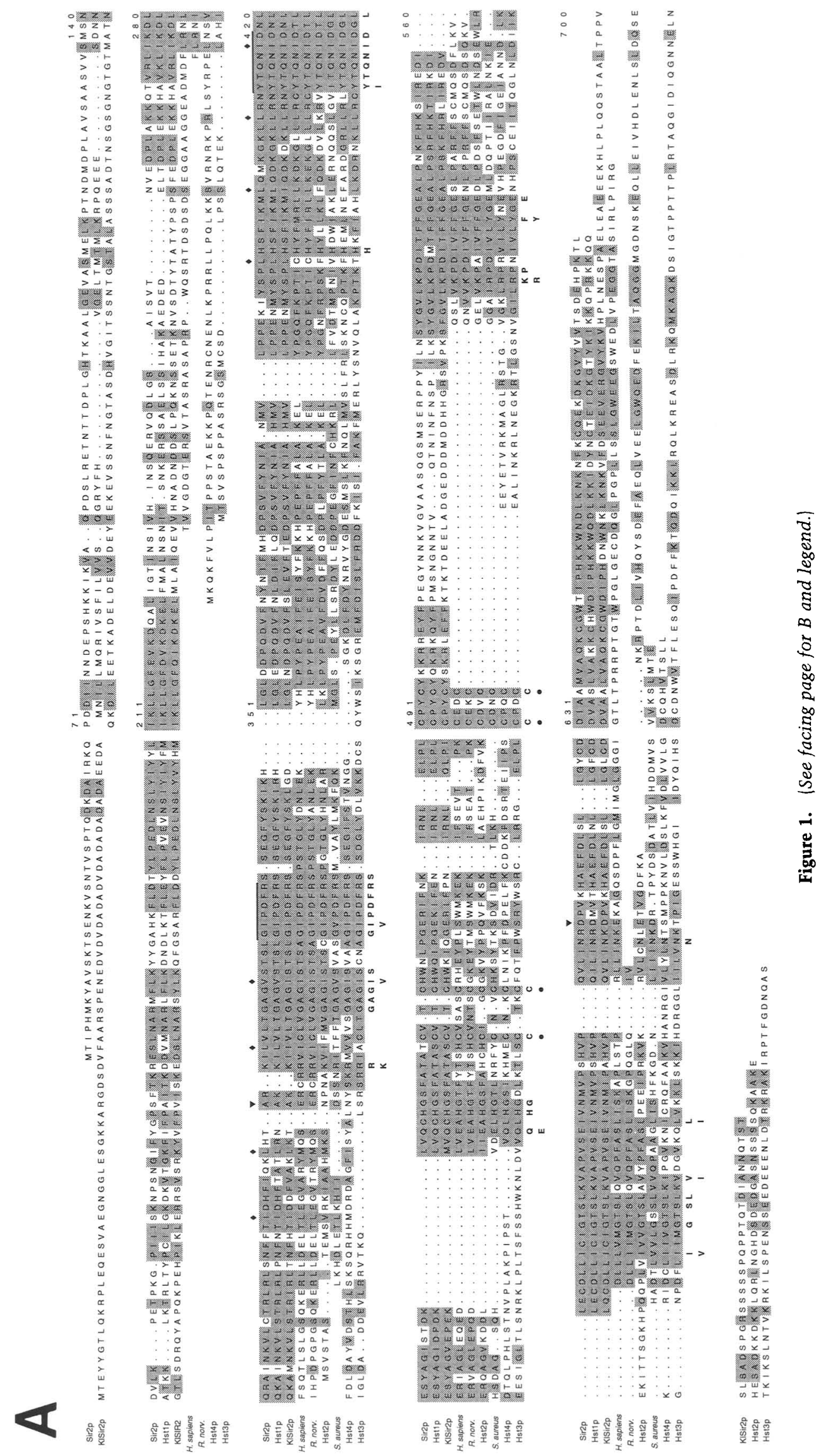


tation. Interestingly, complementation was specific to the MAT $\alpha$ background.

To determine the extent to which HST1 is able to complement the mating defect caused by sir2, standard quantitative mating assays were performed (Sprague 1991). The mating efficiencies of LPY11 and LPY1393 transformed with vector alone or with constructs overexpressing either HST1 or SIR2 were determined. The values presented in Figure 2 represent the average mating efficiencies obtained for at least two independent transformants. The mating efficiency of LPY1393 is $\geqslant 5000$-fold above background when HST1 is overexpressed. However, it is still $\sim 30$-fold lower than LPY1393 transformed with a SIR2 plasmid. Consistent with the qualitative mating results (Fig. 2), no significant complementation of the sir2 mating defect by high copy HST1 was detected in the MATa background.

HST1 could act to restore mating to a sir2 strain directly by functioning in transcriptional silencing or indirectly by influencing other events in the mating pathway. To test whether high copy HST1 suppression of the sir2 mating defect is attributable to partial restoration of transcriptional silencing, a sir2 strain with a SUP3 reporter gene at $H M R$ (LPY1539) was constructed. In this strain, the a information normally present at $H M R$ has been replaced with the gene encoding an amber suppressor tRNA (Schnell and Rine 1986). Because this strain also contains a $\operatorname{trp} 1_{\text {am }}$ mutation, growth on plates without tryptophan reflects transcription of the $S U P 3_{a m}$ gene at hmr (Fig. 2B). The sir2 mutant strain grows well in the absence of tryptophan. In contrast, when these cells are transformed with the HST1 or SIR2 high copy plasmids, they exhibit significantly decreased growth, presumably attributable to restored silencing of the hmr $\Delta:: S U P 3_{a m}$ reporter. Thus, Hstlp not only shares sequence homology with Sir2p, but also has silencing function.

\section{Telomeric silencing in hst mutants}

SIR2 function is necessary for telomeric silencing; therefore, we tested the hst mutants for defects in telomeric position effect. The assay uses a URA3 gene integrated at the subtelomeric $A D H 4$ locus on chromosome IV (ADH4UCA-IV; Aparicio et al. 1991). In wild-type cells the subtelomeric URA3 is expressed stochastically, with a small portion of the population expressing URA3 and a majority of the population silencing its expression. When plated onto 5-fluoro-orotic acid (5-FOA) medium, wild-type cells survive due to this silencing. Strains unable to silence the expression of telomeric $U R A 3$, such as sir2 strains, will not grow on 5-FOA medium. By comparing growth of strains on permissive medium to growth on 5-FOA, one can estimate the extent to which a mutant strain has lost the ability to repress transcription of the telomeric URA3 (Fig. 3). Consistent with previous experiments, sir2 cells were unable to grow on 5-FOA, indicating loss of telomeric silencing. In contrast, the telomeric URA3 gene was repressed comparably in wild-type, hst1, hst2, and hst4 strains (Fig. 3, cf. row $B$ with $C, D$, and $F$, indicating no defect in telomeric silencing in these strains. The hst 3 mutant and wildtype strains had a similar frequency of Foa $^{\mathrm{R}}$ cells, but the hst 3 colonies grew much slower than wild type \{Fig. 3, cf. row B with E). Strikingly, the hst 3 hst 4 double mutant showed significantly greater 5-FOA sensitivity than either single mutant alone /Fig. 3, cf. row $\mathrm{G}$ with $\mathrm{B}, \mathrm{E}$, and $F \mid$, and the number of Foa $^{\mathrm{R}}$ cells is reduced $\sim 100$-fold relative to wild-type. We conclude that HST3 and HST4 play important roles in telomeric silencing. The synergy between hst 3 and hst 4 mutations is explored further in the phenotypic characterization below.

The hst 3 hst 4 double mutant is temperature sensitive, has low viability, and altered cell cycle distribution

We discovered that the hst 3 hst 4 strain is temperature sensitive (Ts) for growth at $37^{\circ} \mathrm{C}$. In contrast to the hst 3 and hst 4 single mutants, and the hst 3 hst 4 double mutant maintaining a centromeric HST3 plasmid, hst 3 hst 4 cells formed colonies at $37^{\circ} \mathrm{C}$ at efficiencies 37 -fold to $>1000$-fold lower than wild type (data not shown). When
B

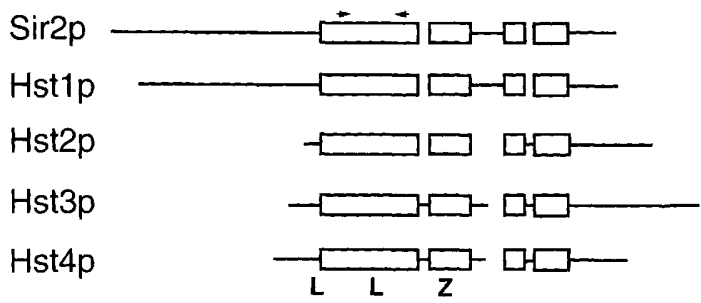

$\begin{array}{lllll} & \text { Sir2p } & \text { Hst1p } & \text { Hst2p } & \text { Hst3p } \\ \text { Hstip } & 63 / 76 & \ldots \ldots & \frac{\% \text { amino acid identity }}{\% \text { amino acid similarity }} \\ \text { Hst2p } & 34 / 56 & 35 / 55 & \ldots \\ \text { Hst3p } & 32 / 54 & 31 / 56 & 25 / 49 & \\ \text { Hst4p } & 29 / 51 & 27 / 50 & 28 / 52 & 35 / 56\end{array}$

Figure 1. Alignment of yeast Hst proteins and related sequences from other species. (A) Amino acid sequence alignment. (O) Cysteine residues of the putative metal binding domain; $(\checkmark)$ leucine residues of the putative leucine zippers; $(\boldsymbol{\nabla})$ boundaries of the core domain; (solid line) sites of degenerate PCR primers used. Shaded residues are those that are identical or similar based on the Blosum 62 matrix; residues in bold below alignment are absolutely conserved in known family members. Alignment was performed using PILEUP; amino acids 477-490 were adjusted manually to align putative metal-binding domain. SIR2 homologs from species other than yeast are included (see Materials and methods). $(B)$ Block diagram of the yeast Hst proteins and Sir2p. The yeast proteins are compared based on the alignment in $A$ with open boxes indicating regions of similarity to Sir2p. Table contains pairwise comparisons between family members calculated by GAP using preset options. The nucleotide sequences of HST1, HST2, and HST3 have been submitted to the GenBank data library under accession nos. U39041, U39063, and U39062, respectively. HST4 is encoded by the cosmid under accession no. Z48784. 
Table 1. Yeast strains

\begin{tabular}{|c|c|}
\hline Strain & Genotype \\
\hline LPY $11^{a}$ & MATa ade2-1 can1-100 his3-11 leu2-3,112 trp1-1 ura3-1 GAL sir2A::HIS3 \\
\hline LPY $1393^{a}$ & MAT $\alpha$ ade2 his3 leu2 sir2A::HIS3 ura3 \\
\hline LPY 142 & MATa his4 \\
\hline LPY143 & MATa his4 \\
\hline LPY1539 & 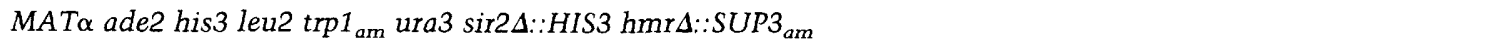 \\
\hline \multicolumn{2}{|c|}{ YPH499,500 and derivatives } \\
\hline YPH499 & 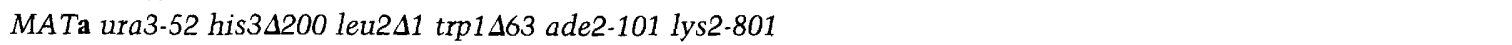 \\
\hline YPH500 & 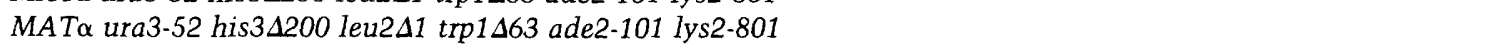 \\
\hline YCB506 & isogenic to YPH499 hst $3 \Delta 3::$ HIS3 hst $4 \Delta 1:: U R A 3$ \\
\hline YCB538 & isogenic to YPH499 hst $3 \Delta 3:: H I S 3$ hst $4 \Delta 1:: U R A 3$ \\
\hline YCB547 & isogenic to YPH500 hst $3 \Delta 3:: H I S 3$ hst $4 \Delta 1:: U R A 3$ \\
\hline $\mathrm{X} 23-9 \mathrm{~A}$ & isogenic to YPH500 hst $4 \Delta 1:: U R A 3$ \\
\hline $\mathrm{X} 23-10 \mathrm{D}$ & isogenic to YPH500 hst $3 \Delta 3:: H I S 3$ \\
\hline YCB405 & isogenic to YPH499 hst3A3::HIS3 \\
\hline YPH279 & 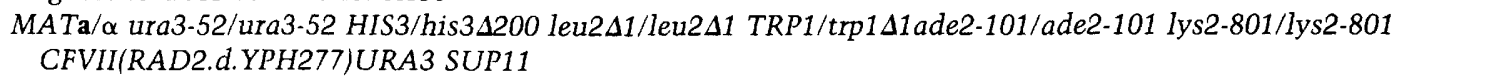 \\
\hline YCB654 & 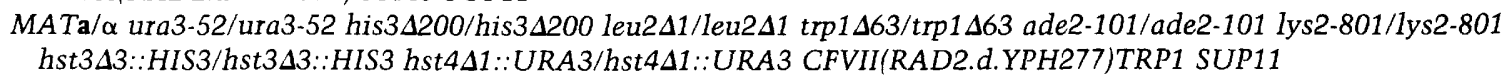 \\
\hline YCB 657 & 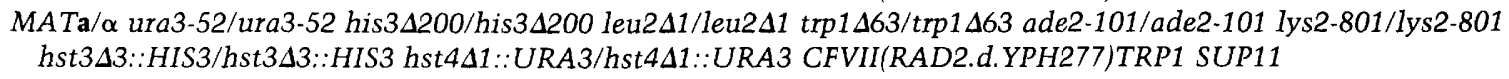 \\
\hline YCB684 & isogenic to YPH500 hst3A3::HIS3 CFVII(RAD2.d.YPH277)TRP1 SUP11 \\
\hline YCB624 & isogenic to YPH499 rad9 $1:: T R P 1$ \\
\hline YCB627 & isogenic to YPH500 hst $3 \Delta 3:: H I S 3$ hst $4 \Delta 1:: U R A 3$ rad $9 \Delta 1:: T R P 1$ \\
\hline YCB632 & isogenic to YPH500 rad9 $1:: T R P 1$ hst $4 \Delta 1:: U R A 3$ \\
\hline YCB633 & isogenic to YPH500 rad9 $\Delta 1:: T R P 1$ hst $3 \Delta 3:: H I S 3$ \\
\hline \multicolumn{2}{|c|}{ FY2 derivatives ${ }^{b}$} \\
\hline YPH681 & 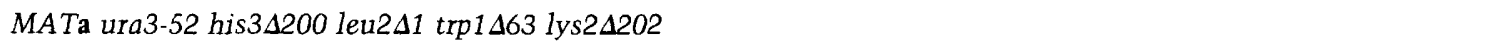 \\
\hline YPH680 & 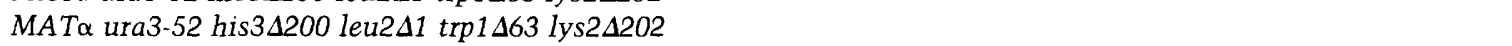 \\
\hline YCB470 & isogenic to YPH681 hst $3 \Delta 3:: T R P 1$ \\
\hline YCB575 & isogenic to YPH681 hst $4 \Delta 1:: T R P 1$ \\
\hline YCB 600 & isogenic to YPH681 hst $3 \Delta 3:: T R P 1$ hst $4 \Delta 1:: T R P 1$ pCAR202 \\
\hline $\mathrm{YCB}^{-} 00^{-}$ & isogenic to YPH681 hst $3 \triangle 3:: T R P 1$ hst $4 \Delta 1:: T R P 1$ \\
\hline YCB601 & isogenic to YPH680 hst $3 \Delta 3:: T R P 1$ hst $4 \Delta 1:: T R P 1$ pCAR202 \\
\hline $\mathrm{YCB} 601^{-}$ & isogenic to YPH 680 hst $3 \Delta 3:: T R P 1$ hst $4 \Delta 1:: T R P 1$ \\
\hline YCB669 & isogenic to YPH681 YCp50 \\
\hline YCB674 & isogenic to YPH681 hst3 $43:: T R P 1$ hst $4 \Delta 1:: T R P 1$ pCAR202 YCp50 \\
\hline YCB $674^{-}$ & isogenic to YPH 681 hst $3 \Delta 3:: T R P 1$ hst $4 \Delta 1:: T R P 1$ YCp50 \\
\hline YCB 647 & isogenic to YPH681 leu2A::TRP1 ADH4::URA3-TEL \\
\hline YCB648 & isogenic to YPH681 hst $1 \triangle 3:: T R P 1$. ADH4::URA3-TEL \\
\hline YCB649 & isogenic to YPH681 hst2 $\triangle 2:: T R P 1$ ade2A1 ADH4::URA3-TEL \\
\hline YCB650 & isogenic to YPH 681 hst $3 \triangle 3: \because T R P 1$ ADH4::URA3-TEL \\
\hline YCB651 & isogenic to YPH 681 hst $4 \Delta 1:: T R P 1$ ADH4::URA3-TEL \\
\hline YCB652 & isogenic to YPH681 sir2 $22:: T R P 1$ ADH4::URA3-TEL \\
\hline YCB653 & isogenic to YPH681 hst3A3::TRP1 hst4A1::TRP1 ADH4::URA3-TEL \\
\hline YCB692 & isogenic to YPH681 ade2::hisG::URA3::hisG \\
\hline YCB693 & isogenic to YPH681 hst $3 \triangle 3:: T R P 1$ ade2::hisG::URA3::hisG \\
\hline YCB694 & isogenic to YPH681 hst4A1::TRP1 ade2::hisG::URA3::hisG \\
\hline YCB695 & isogenic to YPH681 hst $3 \Delta 3:: T R P 1$ hst $4 \Delta 1:: T R P 1$ ade2::hisG::URA3::hisG pCAR202 \\
\hline $\mathrm{YCB} 695^{-}$ & isogenic to YPH681 hst $3 \Delta 3:: T R P 1$ hst $4 \Delta 1:: T R P 1$ ade2::his G::URA3::hisG \\
\hline
\end{tabular}

These strains are derived from W303.

bYPH680 and YPH681 are derived from the FY2 series of strains (Winston et al. 1995).

the hst 3 hst 4 cells that grow at high temperature were restreaked at $37^{\circ} \mathrm{C}$, they maintained their $\mathrm{Ts}^{+}$phenotype (data not shown). Thus, we believe that the variability in frequency of $\mathrm{Ts}^{+}$cells is attributable to sporadic acquisition of suppressors. Because these suppressors can accumulate, many of the experiments performed on hst 3 hst 4 strains were carried out using strain $\mathrm{YCB} 60^{+}$, which carries an HST3 CEN plasmid. The plasmid is segregated just before the experiment, generating $\mathrm{YCB} 60^{-}$, to minimize the accumulation of suppressors.

Microscopically we observed that most of the mutant cells were larger than wild type (Fig. 4), and that a logphase culture of an hst 3 hst 4 strain contained more large-budded cells than wild-type, a phenotype exacerbated at higher temperatures (Fig. 5A). In this large-budded population the daughter cell was often as large as the mother, a form rarely observed in wild-type cultures (Fig. 

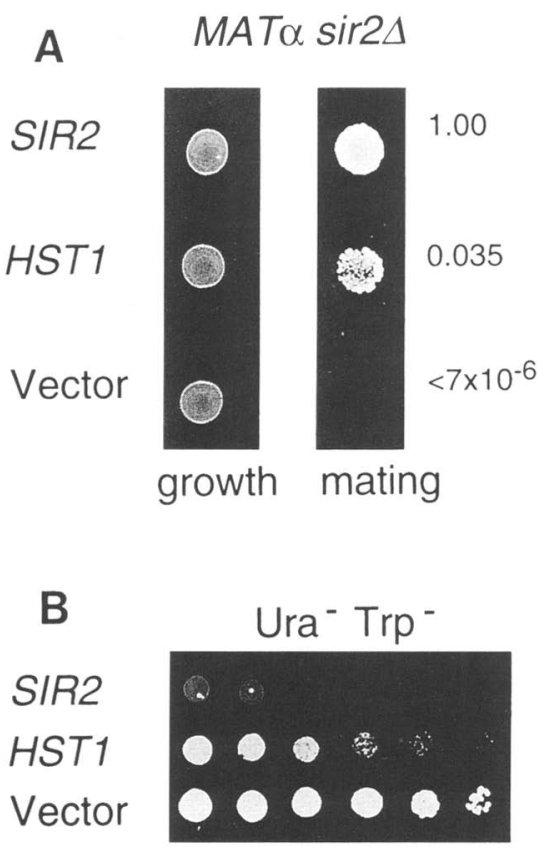
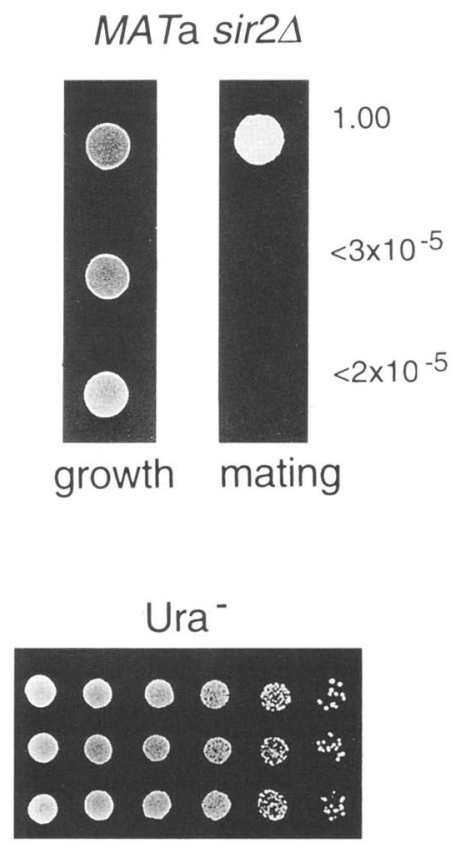

Figure 2. HST1 restores silencing at $H M R$ in sir2 mutants. (A) Increased HST1 gene dosage was tested for ability to complement MATa and $M A T \alpha$ sir2 mating defects. Comparisons to $2 \mu$ vector and a SIR2 $2 \mu$ plasmid are shown. Approximately equal numbers of each transformant were spotted onto SC-Ura to assay growth and onto lawns of the mating testers LPY 142 and LPY143 to assay mating. The mating efficiencies determined by quantitative mating assays are also presented. The values represent the average mating efficiency of at least two independent isolates for each transformant. $(B)$ To determine whether the observed complementation is attributable to restoration of transcriptional silencing at $H M R$, a sir2 strain with a $S U P 3_{a m}$ reporter gene at $H M R$ and a $\operatorname{trp} 1_{a m}$ mutation (LPY1539) was transformed with the $2 \mu$ plasmids. Transformants were assayed for Trp expression by spotting serial dilutions onto SC-Ura-Trp and for growth by spotting on SC-Ura plates.
4). Because cells with large buds indicate a block in mitosis, we examined DNA content by flow cytometry (Fig. 5B). The double mutant strain exhibited a dramatic increase in the number of cells with $\mathrm{G}_{2}$ DNA content. Both single mutants (hst 3 and hst 4 ) are similar to wild type microscopically and by flow cytometry (Fig. 5 ; data not shown). To determine the nuclear morphology of the hst 3 hst 4 mutant strain, we visualized nuclei with the fluorescent dye DAPI. We found that the double mutant showed an increase in the percentage of large-budded cells with the nucleus at or across the neck (Figs. 4 and $5 \mathrm{~A}$ ), but that the percentage of large-budded cells in which the nucleus had divided was similar to wild-type.

\section{$\mathrm{SC} \quad \mathrm{SC}+5-\mathrm{FOA}$}
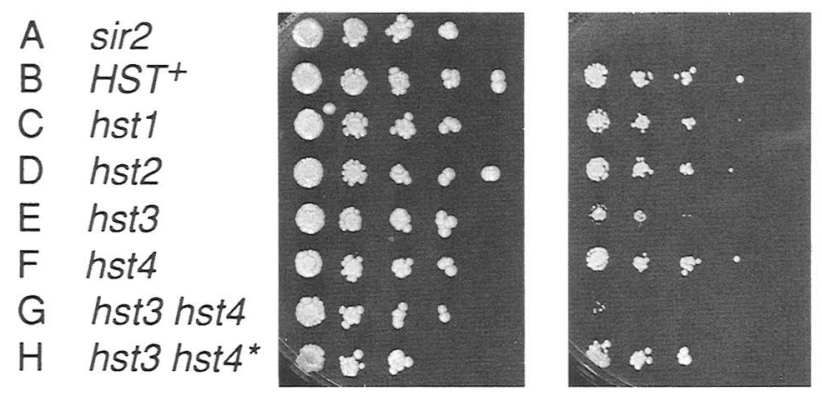

Figure 3. The hst 3 hst 4 mutant shows increased expression of a telomeric URA3 gene. Tenfold serial dilutions were plated onto either SC medium or SC containing $0.1 \% 5$-FOA. Strains used were: (A) YCB652; (B) YCB647; (C) YCB648; (D) YCB649; (E) YCB650; (F) YCB651; (G) YCB653; and (H) YCB600'- Strains A-G contain a telomeric copy of URA3 at $A D H 4$ (ADH4::URA3-TEL), strain $\mathrm{H}$ does not. The 5-FOA sensitivity of the hst 3 hst 4 ADH4::URA3-TEL strain is not attributable to a general 5-FOA supersensitivity because hst 3 hst 4 ura 3 strains lacking telomeric URA3 grow normally on 5-FOA (row $\mathrm{H}$ ).
Therefore, the hst 3 hst 4 strain contains a larger fraction of cells in which the DNA has been replicated but cell division has not occurred.

This accumulation could be the result of a $\mathrm{G}_{2} / \mathrm{M}$ delay, or it could be attributable to the irreversible arrest of a subpopulation of the hst 3 hst 4 mutant cells. To distinguish between these two possibilities we determined the viability of the double mutant cells (Table 2). Whereas the viability of the hst 3 and hst 4 single mutants was similar to wild type, we observed that $44 \%$ of double mutant cells were inviable. This defect was completely corrected by the presence of the HST3 CEN plasmid. Strikingly, when we determined the viability of the hst3 hst 4 large-budded cell population, we found that only $27 \%$ of these cells were viable, as opposed to $89 \%$ in wild type. Thus, it appears that the large-budded cell population in the mutant has two components, a normal component and a larger inviable component lacking in the wild-type population.

By microscopic examination of manipulated cells grown at $37^{\circ} \mathrm{C}$, we determined that the majority of the hst 3 hst 4 large-budded cells that died did so as 2- or 4-cell bodies (of equal size), whereas a few formed microcolonies of 5-20 cells (data not shown). Many of these dead cells were bloated and misshapen. The large-budded cell inviability observed in the hst 3 hst 4 double mutant suggests that the $G_{2} / M$ accumulation observed in the double mutant reflects the existence of a subset of ultimately inviable cells that are arrested at this juncture of the cell cycle, rather than a cell-cycle delay.

\section{The hst3 hst4 rad9 strain is hypersensitive to UV irradiation}

The excess $\mathrm{G}_{2} / \mathrm{M}$ cells observed in the hst 3 hst 4 double mutant strain could result from DNA damage triggering 
$\mathrm{HST}^{+}$

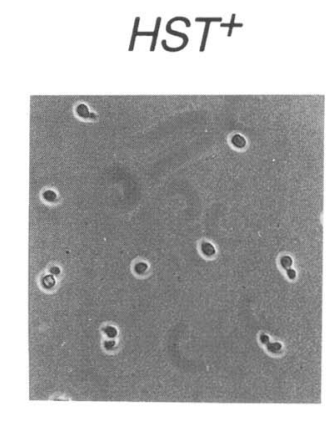

Figure 4. Aberrant morphology of hst 3 hst 4 mutants. Log phase cells of wild type (YPH681) and mutant (YCB600-) strains were visualized by phase contrast. Note increased number of large-budded cells and in creased cell size in mutant culture. Also shown are representative aberrant nuclear and microtubule morphologies of the mutant.

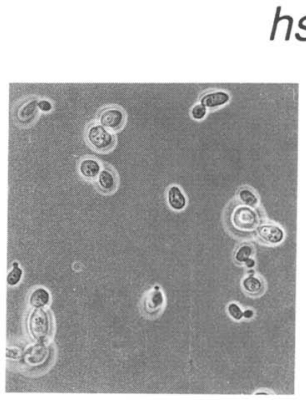

hst3 hst4
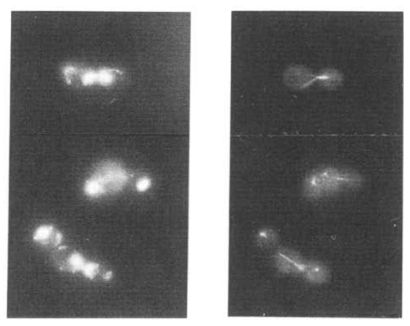

DAPI

$\alpha$-tubulin a $R A D 9$-dependent delay. Thus, we examined the effect of introducing a rad9 mutation into these strains. The prediction of this model is that a triple mutant (hst 3 hst 4 rad9) would lose the $\mathrm{G}_{2} / \mathrm{M}$ accumulation (or lose viability). The triple mutant grows like the hst 3 hst 4 double mutant, and like the hst 3 hst 4 mutant, exhibits a $\mathrm{G}_{2} / \mathrm{M}$ accumulation, indicating that this accumulation is not $R A D 9$ dependent.

We examined the sensitivity of these strains to UV irradiation. The hst 3 hst 4 double mutant showed a modest UV-irradiation sensitivity, similar to that of rad9 alone (Fig. 6). rad9 cells die when UV irradiated because they do not have time to repair DNA damage, but this lethality can be alleviated by inducing a $G_{2} / M$ delay (Weinert and Hartwell 1988). Accordingly, if the increased $\mathrm{G}_{2} / \mathrm{M}$ accumulation in hst 3 hst 4 cells results from a reversible cell-cycle delay at a point similar to a $R A D 9$ checkpoint, in theory hst 3 hst 4 could rescue the UV sensitivity of rad9 cells. When we tested the hst 3 hst 4 rad 9 mutant for UV resistance, we in fact found that the triple mutant is UV hypersensitive relative to either parent (Fig. 6). The synergistic effects of hst mutations and radg are reminiscent of the connection be- tween silencing (SIR3) and DNA repair/checkpoint control (RAD7) pathways (Paetkau et al. 1994).

\section{hst 3 hst 4 mutants display increased chromosome loss and recombination}

Many mutant strains that exhibit an accumulation of $\mathrm{G}_{2} / \mathrm{M}$ cells lose chromosomes at an increased rate /Gerring et al. 1990; Doheny et al. 1993). We tested the hst3 hst 4 double mutant for chromosome stability using an artificial chromosome and a visual ploidy assay (Spencer et al. 1990; Shero et al. 1991). Our analysis made use of two marked chromosome fragments (derived from two different native yeast chromosomes), which result in a white colony in an ade2 background; colonies lacking the marked fragment are red (Hieter et al. 1985). Using this assay we observed that the hst 3 hst 4 strain showed high-frequency sectoring with both chromosome fragments. In addition, the hst 3 mutant exhibited a modest increase in sectoring, whereas the hst 4 mutant was indistinguishable from wild type.

The frequency of loss of one such fragment was calculated in diploid strains in which the dosage of the frag-
Figure 5. Altered cell cycle distribution in hst3 hst 4 mutant cells. (A) Morphological analysis. Log phase cultures in YPD were fixed and stained with DAPI to visualize nuclei. Similar results were obtained in two separate experiments. The temperature shift experiment was performed by collecting log phase cells from $30^{\circ} \mathrm{C}$ YPD cultures, resuspending them in prewarmed $37^{\circ} \mathrm{C} Y P D$, and incubating them for $4.5 \mathrm{hr}$ at $37^{\circ} \mathrm{C}$ before analysis. At $37^{\circ} \mathrm{C}$, a number of large-budded mutant cells appear to have budded a third time, and in some cases a fourth (data not shown). Strains used were YPH499 and YCB506. (B) FACScan analysis. Log phase cultures were analyzed by flow cytometry. Number of cells is shown on the y-axis, and fluorescent intensity of emitted light is shown on the $\mathrm{x}$-axis. The slight $\mathrm{x}$-axis shift seen in the double mutant is likely caused by its increased cell size. Strains used were YPH499, YCB538, X23-10D, and X23-9A.
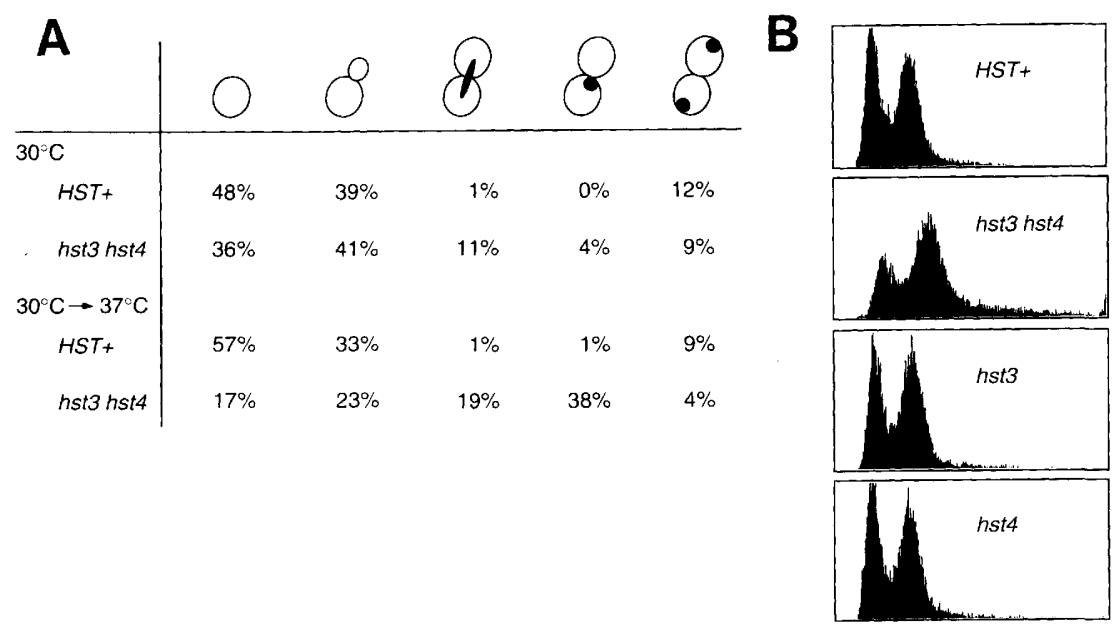
Table 2. Viability measurements

\begin{tabular}{llrrr}
\hline Strain & Relevant genotype & $\begin{array}{l}\text { Percent viability } \\
\text { (all cells) }\end{array}$ & $\begin{array}{l}\text { Percent viability } \\
\text { (large-budded cells) }\end{array}$ & $\begin{array}{l}\text { Percent } \\
\text { large-budded cells }\end{array}$ \\
\hline YPH680 & HST & 89 & 26 \\
YCB470 & hst3 & 89 & 82 & 18 \\
YCB575 & hst4 & 100 & 100 & 14 \\
YCB601 & hst3 hst4 [HST3 CEN] & 88 & 79 & 18 \\
YCB601- & hst3 hst4 & 44 & 27 & 56 \\
\hline
\end{tabular}

${ }^{a}$ Viability was determined by micromanipulation and growth of individual cells on YPD at $30^{\circ} \mathrm{C}$.

befined as cells in which daughter cell is $>50 \%$ the size of mother cell.

ment can be assessed. A single copy of the chromosome fragment in an ade2-101 homozygote results in pink colonies, whereas colonies containing two (or more) copies of the fragment are white. This assay allows the rate of chromosome missegregation to be calculated, and differentiates between chromosome loss (1:0 segregation) and chromosome nondisjunction (2:0 segregation) events. A 1:0 missegregation event in the first division results in a half red/half pink sectored colony; similarly, a half red/ half white sectored colony is the result of a 2:0 missegregation event. The hst 3 hst $4 /$ hst 3 hst 4 double mutant exhibited a dramatic chromosome missegregation rate of 35 -fold above the wild type (Table 3). Nondisjunction events (46-fold elevated) were somewhat more frequent than chromosome loss events (25-fold elevated). The hst $3 /$ hst 3 homozygote showed a modest missegregation rate increase of less than twofold over the wild-type homozygote (Table 3). We also examined the rate of loss of the small circular plasmid YCp50 in wild-type and mutant strains. We found that in the hst 3 hst 4 mutant the rate of plasmid loss was elevated 2.6-fold above wild type (Fig. 7). Thus, the stability of both plasmids and chromosome fragments is reduced in the hst 3 hst 4 mutant, but the much larger chromosome fragments show a more stringent requirement for the HST genes.

Homologous recombination frequency provides another insight into genomic stability. We examined recombination frequency using two assays. The first uses an $h s t 3$ hst $4 /$ hst 3 hst 4 CAN $1^{S} /$ can $1^{R}$ strain. Because the $C A N 1^{S}$ allele is dominant, it renders diploid cells sensitive to canavanine. If the $C A N 1^{S}$ allele is lost, generally by gene conversion of the $C A N 1^{S}$ allele to $\operatorname{can} 1^{R}$, cells become resistant to canavanine $\left(\mathrm{Can}^{\mathrm{R}}\right)$. The hst 3 hst 4 mutants exhibited an increase in the frequency of $\mathrm{Can}^{\mathrm{R}}$ cells relative to wild type. $\mathrm{Can}^{\mathrm{R}}$ cells can result from loss of chromosome $\mathrm{V}$, leaving the cell with a single can $1^{R}$ allele, or from recombinational homozygosis. Because the observed increase in frequency in Can ${ }^{\mathrm{R}}$ cells is complicated by the fact that the hst 3 hst 4 mutant has a chromosome segregation defect, we designed a second assay using the $A D E 2$ disrupter $\mathrm{p} \triangle \mathrm{ADE} 2$ (Aparicio et al. 1991). When integrated at $A D E 2$, this construct replaces part of $A D E 2$ with direct repeats of hisG flanking URA3. We integrated this ade2::hisG::URA3::hisG construct into our mutant haploid strain, selected $\mathrm{Ura}^{+}$cells, and allowed the resultant Ade ${ }^{-}$(pink) cells to grow on medium containing uracil. We then asked how many cells had become Foa $^{\mathrm{R}}$ by recombination between the repeated his $G$ sequences. We observed a fivefold increase in mitotic recombination in hst 3 hst 4 strains relative to wild type, and more modest increases in the single mutants (Table 4). Thus, in addition to playing roles in silencing, proteins of the Sir $2 p$ family affect chromosome stability and recombination, indicating a more general role in chromatin metabolism.

\section{Discussion}

The SIR2 gene family

Members of the yeast SIR2 gene family we have discovered range from $29 \%$ to $63 \%$ identical to Sir 2 p overall at

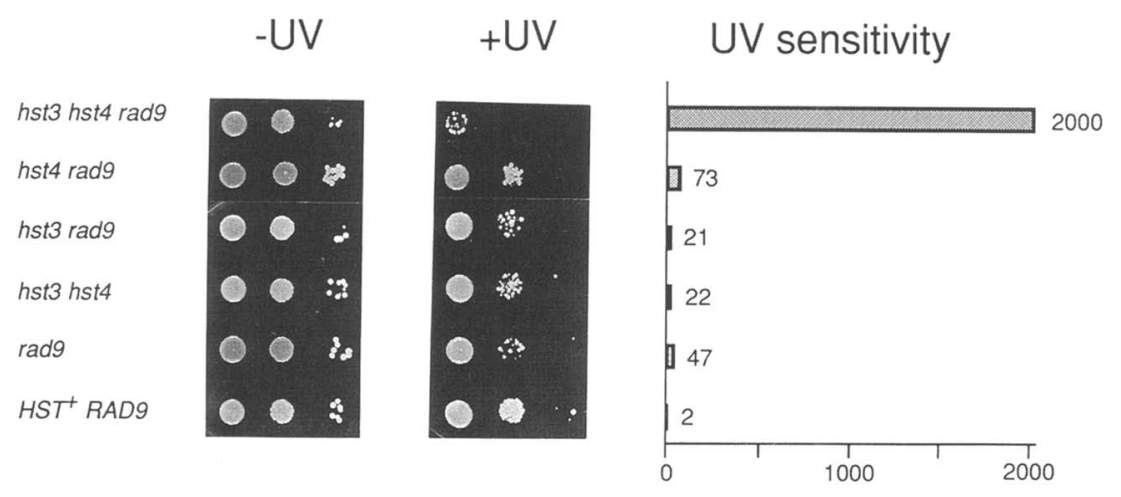

Figure 6. UV sensitivity of the hst3 hst4 double mutant and hypersensitivity of the hst 3 hst 4 rad 9 triple mutant. Five microliters of 100-fold serial dilutions were spotted onto YPD plates and either UV irradiated with $80 \mathrm{~J} / \mathrm{m}^{2}$ using a Stratalinker (Stratagene; setting of $\left.80 \mu \mathrm{J} \times 100 / \mathrm{cm}^{2}\right)$ immediately and again $12 \mathrm{hr}$ later ( UV) (Weinert et al. 1994), or left unirradiated (-UV). Plates were wrapped in foil to prevent photoreactivation and incubated at $30^{\circ} \mathrm{C}$. The bar graph depicts the results numerically as UV sensitivity (number of cells exposed to UV/number of cells surviving UV exposure). Strains used, from top to bottom, were YCB627, YCB632, YCB633, YCB547, YCB624, and YPH500. 
Table 3. Chromosome fragment loss rates

\begin{tabular}{|c|c|c|c|c|c|}
\hline Strain & Genotype & $\begin{array}{l}\text { Chromosome } \\
\text { mis-segregation rate }\end{array}$ & Percent 1:0 events ${ }^{a}$ & Percent $2: 0$ events $^{\mathrm{b}}$ & $\begin{array}{l}\text { No. of colonies } \\
\text { with } 1 \mathrm{CF}^{\mathrm{c}}\end{array}$ \\
\hline YPH 279 & $H S T^{+} / H S T^{+}$ & 0.06 & 0.03 & 0.03 & $29046^{d}$ \\
\hline \multirow{3}{*}{ YCB654 } & $\begin{array}{l}\text { hst } 3 / \text { hst } 3 \\
\text { hst } 3 \text { hst } 4 / \text { hst } 3 \text { hst } 4\end{array}$ & 0.11 & 0.053 & 0.053 & 1900 \\
\hline & a & 1.41 & 0.28 & 1.13 & 708 \\
\hline & $\mathrm{b}$ & 2.48 & 1.14 & 1.34 & 968 \\
\hline \multirow[t]{3}{*}{ YCB657 } & hst 3 hst $4 /$ hst 3 hst 4 & & & & \\
\hline & a & 2.09 & 0.91 & 1.18 & 1102 \\
\hline & $\mathrm{b}$ & 2.5 & 0.66 & 1.84 & 760 \\
\hline Mean \pm S.D. & hst 3 hst $4 /$ hst 3 hst 4 & $2.12 \pm 0.51$ & $0.75 \pm 0.37$ & $1.37 \pm 0.32$ & \\
\hline
\end{tabular}

The above strains were plated for single colonies, and half-sectored colonies (at least $50 \%$ red) were counted. YCB654 and YCB657 are isogenic diploids, and $\mathrm{a}$ and $\mathrm{b}$ denote duplicate experiments for each. The final line shows the mean of the four hst 3 hst $4 / h s t 3$ hst 4 experiments and indicates the standard deviation. Number of half sectored colonies scored: YCB654a (10); YCB654b (24); YCB657a (23); YCB657b (19); YCB405 × YCB684 (2).

${ }^{a}$ Number of red/pink half-sectored colonies divided by total colonies with one chromosome fragment (CF).

${ }^{b}$ Number of red/white half-sectored colonies divided by total colonies with one CF.

'The total colonies with one CF.

${ }^{d}$ From Gerring et al. (1990).

the amino acid level with most variation occurring in the length and sequence of their amino and carboxyl termini. All the HSTs contain an even more highly conserved core region that includes several absolutely conserved motifs of unknown function, four completely conserved cysteines hypothesized to form a zinc finger and a partially conserved putative leucine zipper domain. The zinc finger and leucine zipper motifs suggest that these proteins may form complexes with other pro-

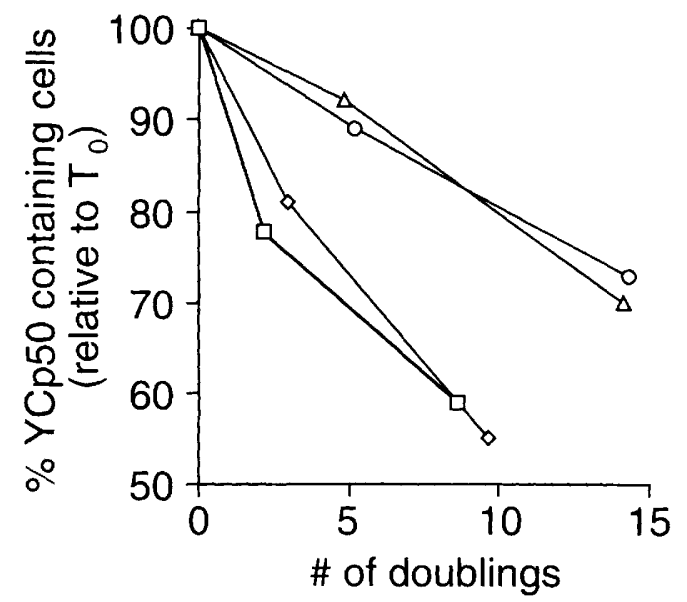

Figure 7. Elevated loss of a CEN plasmid in hst 3 hst 4 mutant cells. Mutant strains show an average loss rate 2.6-fold greater than wild-type strains (Dani and Zakian 1983). Log phase cultures selecting for the CEN plasmid YCp50 were diluted into YPD and grown nonselectively for the number of generations indicated. Cells were plated on YPD and selective plates to determine the number of cells that had lost the plasmid. The results depict two YCp50 transformants each for YCB674- $\square$ and $\diamond \mid$ and YCB669 $\mid O$ and $\triangle \mid$ normalized to percentage of plasmid-bearing cells in selective cultures at time $0184 \%$ and $89 \%$ for $\mathrm{HST}^{+}, 73 \%$ and $81 \%$ for hst3 hst 4 ). teins and bind to DNA. Consistent with this is the hypersensitivity to DNA-targeting drugs of sir2 mutants in K. lactis (Chen and Clark-Walker 1994) and the UV hypersensitivity reported here. However, there is as yet no evidence that Sir2p or the Hst proteins bind DNA.

\section{HST genes have silencing functions}

The HST-encoded proteins exhibit both remarkable sequence similarity to Sir2p as well as functional overlap. Hstlp is Sir2p's closest relative. It is $63 \%$ identical to Sir $2 p$ overall and $82 \%$ identical in the conserved core. HST1 is not normally required for silencing at either the $H M$-type loci or telomeres as an hst1 strain mates and maintains normal levels of telomeric repression. However, we have shown that, in a novel instance of matingtype specific suppression, overexpression of HST1 restores significant mating competence to a MAT $\alpha$ sir2 strain. That this is attributable to a restoration of silencing at $H M R$ is demonstrated by the reduction in expression of $H M R$-located $S U P 3_{a m}$. A possible explanation for $H S T 1$ 's $\alpha$-specific complementation of the sir2 mating defect may lie in the fact that repression is reestablished more readily with an $H M R$ than with an $H M L$ silencer element (Shei and Broach 1995) and that the function of HST1 may be to facilitate the establishment of repression at $H M R$.

Significantly, we have shown that expression of a telomeric URA3 gene is derepressed in an hst 3 hst $4 \mathrm{mu}-$ tant strain. Although not as extreme as the sir2 telomeric silencing defect, this demonstrates that telomeric silencing is perturbed in the double mutant. This derepression appears to be specific for the telomeres because hst 3 hst 4 mutant strains mate as well as wild-type. In contrast, sir2 mutants are completely nonmating. The observed telomeric, but not $H M$ loci, derepression may be attributable to competition for silencing factors, with 
priority given to silencing the $H M$ loci (Renauld et al. 1993|, or may reflect overlapping but distinct specificities among the members of this gene family.

These experiments demonstrate that at least two of the HST family members have roles in transcriptional silencing, a third can participate in silencing processes, and that these genes can function at both the HM loci and telomeres.

\section{hst 3 hst 4 mutants arrest with undivided nuclei}

HST3 and HST4 encode proteins of a subfamily distinct from Sir2p and Hstlp and are each other's closest relatives (Fig. 1B). HST3 and HST4 appear to encode redundant functions as double mutants have a number of phenotypes that are much less severe or lacking in hst 3 and hst 4 single mutants. A striking phenotype of hst 3 hst 4 double mutant strains is the accumulation of large-budded cells with an undivided nucleus localized to the bud neck.

The cell cycle defects in the hst 3 hst 4 double mutant suggest several possible explanations for the observed derepression of the telomeric regions in these strains. First, a similar accumulation of cells in $\mathrm{G}_{2} / \mathrm{M}$ has been observed in a histone $\mathrm{H} 4$ mutant with multiple lysine to glutamine substitutions in the amino terminus (Megee et al. 1995). These mutants may mimic the constitutively acetylated, transcriptionally active state. Hypoacetylation of histones correlates with increased DNA compaction and thus transcriptional silencing (Lin et al. 1989). If like Sir2p, Hst3p and Hst4p are involved in modulating the levels of histone acetylation or deacetylation, hst 3 hst 4 null mutations could cause increased levels of histone acetylation and thus increased transcription. A second possibility is that the longer $G_{2} / M$ phase of the cell cycle may allow altered assembly of chromatin into a transcriptionally active state (Aparicio and Gottschling 1994).

The cell cycle defects could also be interpreted to suggest that loss of the silencing activities of HST3 and HST4 triggers a checkpoint that halts the cell at the $\mathrm{G}_{2} / \mathrm{M}$ boundary. Cells unable to repair the problem would account for the decreased viability; those able to correct the problem would, after a delay, continue on into the next cycle. It is possible that a few cells might slip through the checkpoint and continue without properly correcting the defect, accounting for the nonviable cells remaining in the population. However, our data more strongly suggest that the accumulation of $G_{2} / M$ phase cells results from a subpopulation that are ultimately inviable-most of the large-budded cells cannot form colonies and many of them fail to divide at all, continuing to grow (but not divide) until they eventually burst. What might send this subpopulation of hst 3 hst 4 cells down this pathway of inexorable destruction? Possible primary defects we have considered, several of which have been observed previously in sir mutants, are DNA damage, improperly replicated DNA, incomplete chromosome condensation, or inability to properly assemble mitotic machinery.
Table 4. Altered recombination in hst mutants

\begin{tabular}{lll}
\hline Strain & $\begin{array}{l}\text { Relevant } \\
\text { genotype }\end{array}$ & $\begin{array}{l}\text { Recombination } \\
\text { frequency }\end{array}$ \\
\hline YCB692 & HST $^{+}$ & $1.7 \pm 0.45 \times 10^{-3}$ \\
YCB693 & hst3 & $3.7 \pm 0.24 \times 10^{-3}$ \\
YCB694 & hst4 & $2.2 \pm 0.33 \times 10^{-3}$ \\
YCB695 $^{-}$ & hst3 hst4 & $8.7 \pm 1.8 \times 10^{-3}$ \\
\hline
\end{tabular}

${ }^{\mathrm{a} C a l c u l a t e d}$ as $\mathrm{Foa}^{\mathrm{R}}$ cells/total cells; mean of at least three independent determinations.

\section{HST3 and HST4 are required for genomic integrity}

Previously, SIR functions have been associated with chromosome stability and positioning. For example, sir 4 mutants lose chromosomes at a fourfold higher rate than wild type, and sir 3 and sir 4 mutants exhibit telomeric shortening and aberrant perinuclear localization (Palladino et al. 1993|. In comparison, hst3 hst4 double mutants display a 35 -fold higher chromosome missegregation rate. Interestingly, the rate of chromosome nondisjunction is twice that of chromosome loss, suggesting a defect in sister chromatid separation during anaphase. It is unlikely that these phenotypes result simply from microtubule defects, because these strains show normal sensitivity to benomyl. Another possibility is disruption of centromeric function as mutations affecting the function of the centromere display $a_{\mathrm{G}_{2}} / \mathrm{M}$ delay and a high rate of chromosome fragment loss. We have observed aberrant mitotic spindles in the hst 3 hst 4 mutant, consistent with aberrant chromosomal condensation or structure in the hst 3 hst 4 mutant. It has been shown that cells displaying reduced chromosomal condensation before anaphase (Hirano et al. 1986) as well as other chromosome segregation defects (Doheny et al. 1993), lose chromosomes and exhibit a "Cut" phenotype in which the nucleus appears pinched in the middle; we observed the Cut phenotype in the hst 3 hst 4 mutant (see Fig.4). In Drosophila, the mus-101 mutant, like the hst 3 hst 4 double mutant, is Ts lethal, has cell cycle and DNA repair defects, a chromosome segregation defect, and is defective in heterochromatin condensation (Gatti et al. 1983). Taken together, these results suggest that loss of silencing and/or chromosome condensation can lead to cell cycle, repair, and chromosome stability defects in diverse organisms.

Because mutations in sir2 have been demonstrated to cause elevated levels of both mitotic and meiotic recombination in the rDNA repeats, we tested recombination frequencies in the hst 3 hst 4 double mutant background. Using two different assays, we have shown that hst 3 hst 4 strains exhibit a fivefold increase in general mitotic recombination. This is similar to the fourfold increase in mitotic recombination observed in sir 3 strains (Palladino et al. 1993) and in contrast to the sir2 mutants whose effects on recombination appear specific for the highly repetitive rDNA (Gottlieb and Esposito 1989).

The accumulation of large budded cells with a $\mathrm{G}_{2}$ DNA content observed in hst 3 hst 4 double mutant strains sug- 
gests that a checkpoint may have been triggered leading to an arrest in $\mathrm{G}_{2} / \mathrm{M}$. In fact, the evidence described above points toward activation of a DNA-damage checkpoint. Thus, we tested whether the arrest caused by mutations in hst 3 and hst 4 was RAD9 dependent. Although no decrease in viability was observed in the hst 3 hst 4 rad9 triple mutant, these cells were hypersensitive to UV irradiation. These results suggest that the HST genes and $R A D 9$ are in parallel pathways that maintain genome integrity.

Finally, we have obtained evidence that chromosome maintenance is grossly defective in hst 1 hst 2 hst 3 hst 4 strains, both in terms of chromosome number and structure. We have observed a number of defects in the electrophoretic karyotypes of these strains and their $\mathrm{Ts}^{+}$revertants (C. Brachmann, E. Cameron, and J. Boeke, unpubl.). We conclude that the Hst proteins are critical for maintaining genomic integrity.

\section{Potential functions of the gene family}

We propose that at least some of the new members of the SIR2 gene family may be involved in the silencing of specific genomic regions, different from known silent loci. Each HST gene may have evolved specificity for one or more genomic regions; thus, Sir $2 p$ is essential for the "silencing complex" specific to the HM loci and telomeres. Although still somewhat speculative, there is evidence indicating that silencing complexes with altered specificities might include SIR1, SIR3, and SIR4. Recently, a semidominant mutant of SIR4 encoding a carboxy-terminally truncated protein has been described that delays aging in yeast (Kennedy et al. 1995). This mutant has lost the ability to participate in silencing at the $H M$ loci and telomeres, leading to their derepression. In addition, expression of only the carboxyl terminus of Sir4p extends the life span of wild-type cells, but reduces silencing at $H M$ loci and telomeres. Together these experiments suggest that the carboxyl residues of Sir $4 p$ are responsible for recruiting the silencing complex to the $H M$ loci and telomeres, and that when Sir $4 p$ can no longer recognize these regions, the complex is free to more tightly silence other parts of the genome. (An interesting requirement of this model is that silencing at the known loci be dominant to silencing at other regions of the genome.) We propose that Sirlp, Sir $3 p$, and Sir $4 p$ may be components of a single silencing complex, the specificity of which is provided by interaction with a SIR2 family member or members. Thus, if the HST genes are involved in silencing gene expression through altered chromatin structure, it is likely that additional, extensive genomic regions are silenced and structurally altered. Perturbations of such chromosomal structures may account for the genomic instability we have observed.

Our data show that HST1 can function in transcriptional silencing at $H M R$, and that hst 3 hst 4 mutants have a telomeric silencing defect. Moreover, hst3 hst4 mutants display a number of phenotypes suggestive of defects in chromatin structure. Three general hypotheses may account for the observed results.

The first hypothesis linking the silencing functions of these genes to the mutant phenotypes is that the HST genes silence other genes; expression of these leads to a $\mathrm{G}_{2} / \mathrm{M}$ delay and the other phenotypes described here. Such a scenario requires that these genes must normally function in a pathway other than vegetative growth (e.g., sporulation, diploid-specific growth, maintenance during starvation, pseudohyphal growth, or growth using unusual nutrients), because their expression in a logarithmically growing population leads to increased cell death.

A second possibility is that the hst mutant phenotypes are attributable to aberrant DNA replication. It is well established that silenced regions replicate late in S phase (McCarroll and Fangman 1988; Fangman and Brewer 1992; Ferguson and Fangman 1992). Loss of silencing caused by the deletion of the HST genes could be associated with an increased number of chromosomal regions replicating early. Alternatively, if the HST genes play a role in "silencing" replication origins after they have fired, there might be some overreplication in the mutant. Altered replication timing may lead to improperly replicated DNA, and ultimately impair the chromosome segregation machinery. The double mutant phenotypes are generally consistent with such a hypothesis, although we have no evidence for a large population of cells with incompletely replicated DNA. Thus, if aberrant replication occurs in the mutant, it may be a relatively subtle defect.

A third hypothesis is that loss of silencing caused by disruption of the HST genes results in a change in chromosomal structure significant enough to cause $a \mathrm{G}_{2} / \mathrm{M}$ delay, chromosomal missegregation, and hyper-recombination. It is clear that silenced regions are structurally distinct from their transcriptionally active counterparts; silenced DNA is inaccessible to the UV-damage repair machinery (Terleth et al. 1989), nucleases (Loo and Rine 1994), and methylation by heterologous DNA methylation enzymes (Gottschling 1992; Singh and Klar 1992). Although there is no cytological evidence for heterochromatic regions in yeast, the correlation of heterochromatin with transcriptional repression in other organisms (PEV in Drosophila, $\mathrm{X}$ inactivation in mammals) and the importance of histones $\mathrm{H} 3$ and $\mathrm{H} 4$ in the process of silencing (Kayne et al. 1988; Johnson et al. 1990; Megee et al. 1990; Park and Szostak 1990; Thompson et al. 1994) suggest that the $H M$ loci (and telomeres) in yeast may be defined functionally as heterochromatic. Gross alterations of chromosomal architecture, such as those that might result from derepression of a large number of normally silenced regions (as may be the case in the hst mutants), could have a deleterious effect on the cycling cell. The phenotypic similarities of the constitutively active H4 mutant (Megee et al. 1995) and the hst3 hst4 mutant support the notion that global loss of silencing leads to cell cycle and chromosome segregation defects. Such altered chromatin might be recognized as aberrant by a cell cycle regulator, or the altered chromatin might 
be damaged during attempts to segregate sister chromatids, or might present some physical impediment to proper segregation. Any one of these changes might lead to $G_{2} / M$ arrest and ultimately, to cell death.

\section{SIR2 homologs from bacteria to humans}

Transcriptional silencing phenomena are observed in organisms from bacteria to humans and are important for such critical functions as bacterial virulence (Dagberg and Uhlin 1992) and X-chromosome inactivation (Migeon 1994). Although mechanisms of silencing and heterochromatin-mediated repression are arguably similar (Laurenson and Rine 1992; Weiler and Wakimoto 1995), little molecular overlap beyond histones has been observed previously. Our discovery of the SIR2 family with homologs from S. aureus, Arabidopsis thaliana, S. pombe, C. elegans, Mus musculus, $R$. norvegicus, and $H$. sapiens suggests that these genes may fulfill an important conserved function. By extension, the existence of HSTs in such diverse organisms also suggests that SIRdependent silencing mechanisms may be used to regulate gene expression and genomic stability from simple to complex organisms.

\section{Materials and methods}

\section{HST gene identification and cloning}

HST1 was identified by several techniques including (1) a data base search in which the Sir2 protein sequence was searched against the translated GenBank and EMBL data bases using the BLAST network service and (2) low stringency blot hybridization. A region of homology to Sir2p was found within the $3^{\prime}$ flanking sequence of NUF2. A genomic clone containing the HST1 gene (pGAD42C) and some sequence data were independently provided by $\mathrm{C}$. Yan/T. Mélèse /Columbia University, $\mathrm{NY}$ ) and G. Schlenstedt/P. Silver (Dana-Farber Cancer Institute, Boston, MA|. An HST1-specific probe hybridizes to Olson $\lambda$ clone 3083 on chromosome XV.

HST2 was originally identified in a two-hybrid screen designed to find interactors with TYA protein. The plasmid p113-2 was obtained from the library of partial Sau3A-digested yeast genomic DNA generously provided by P. Bartel and S. Fields (Chien et al. 1991), and contained the complete HST2 gene fused six codons upstream of its ATG to the GAL4 DNAbinding domain. Subsequent screens of a genomic library (C. Connelly and P. Hieter, unpubl.) allowed isolation of plasmid pCAR135 containing intact HST2. An HST2-specific probe hybridized to Olson cosmid 9901 on chromosome XVI.

By aligning Sir2p, K. lactis Sir2p, S. aureus Sir2p, and Hst2p, we defined conserved sequence motifs, allowing degenerate primer design (Fig. 1A). To identify HST3, we used degenerate PCR on genomic DNA from sir2 hst1 hst2 strain YCB240 with primers JB710 (GGNRTNCCNGAYTTY[A/C]G) and JB708 (RTClA/G/T]ATRTTYTGNGTRTA). Touchdown PCR conditions were as follows: $1 \mathrm{~min}$ at $94^{\circ} \mathrm{C}, 30 \mathrm{sec}$ at $54^{\circ} \mathrm{C}, 30 \mathrm{sec}$ at $72^{\circ} \mathrm{C}$ ] for 2 cycles, $1 \mathrm{~min}$ at $94^{\circ} \mathrm{C}, 30 \mathrm{sec}$ at $50^{\circ} \mathrm{C}, 30 \mathrm{sec}$ at $72^{\circ} \mathrm{C}$ for 2 cycles, $1 \mathrm{~min}$ at $94^{\circ} \mathrm{C}, 30 \mathrm{sec}$ at $46^{\circ} \mathrm{C}, 30 \mathrm{sec}$ at $72^{\circ} \mathrm{C}$ for 2 cycles, $1 \mathrm{~min}$ at $94^{\circ} \mathrm{C}, 30 \mathrm{sec}$ at $42^{\circ} \mathrm{C}, 30 \mathrm{sec}$ at $72^{\circ} \mathrm{C}$ for 2 cycles, $1 \mathrm{~min}$ at $94^{\circ} \mathrm{C}, 30 \mathrm{sec}$ at $38^{\circ} \mathrm{C}, 30 \mathrm{sec}$ at $72^{\circ} \mathrm{C}$ for 2 cycles, $1 \mathrm{~min}$ at $94^{\circ} \mathrm{C}, 30 \mathrm{sec}$ at $48^{\circ} \mathrm{C}, 30 \mathrm{sec}$ at $72^{\circ} \mathrm{C}$ for 25 cycles, $5 \mathrm{~min}$ at $72^{\circ} \mathrm{C}$. The $\sim 240$-bp band was gel purified, ethanol precipitated, and treated with T4 polymerase to create a blunt end (a process referred to as blunting) and cloned into pBSII SK + (Stratagene) linearized with EcoRV. The insert fragment from one subclone (pCAR187) was used to probe a genomic library (C. Connelly and P. Hieter, unpubl.). Three plasmids with overlapping inserts (pCAR192, pCAR195, and pCAR196) were obtained. The HST3 probe hybridized to Olson $\lambda$ clone 4170 on chromosome XV.

To identify HST4, we performed degenerate PCR as described above on genomic DNA from strain YCB388 (sir2 hst1 hst2 hst3) and cloned a 240-bp product. The HST4 probe hybridizes to Olson $\lambda$ clones 4040 and 6708 on chromosome IV.

\section{DNA sequencing}

DNA sequences were determined on both strands for HST1, $H S T 2$, and HST3 using a recently developed in vitro transposonmediated sequencing technique (Devine and Boeke 1994) in conjunction with manual or ABI automated sequencing. HST1 and HST3 were sequenced using the artificial trimethoprimresistance transposon AT-2 (Devine and Boeke 1994), which was inserted into the plasmids pGAD42C and pCAR206 carrying $2.2-\mathrm{kb} H S T 1$ and $1.9-\mathrm{kb}$ HST3 inserts, respectively. HST2 was sequenced using a progenitor artificial transposon closely resembling AT-2 but with slightly different termini, constructed by PCR, and integrated into pCAR143, carrying a 4.5 $\mathrm{kb}$ HST2 insert. Approximately 500-5000 independent artificial transposon recombinants were generated for each plasmid target using Ty1-VLP integrase in vitro. Ampicillin/trimethoprim-resistant recombinants (30-60 per plasmid target) were screened by restriction mapping to identify transposon insertions in the respective HST inserts. Miniprep DNA from recombinants chosen for sequence analysis were treated with $10 \mu \mathrm{g} /$ $\mathrm{ml}$ of RNase A for $30 \mathrm{~min}$ at $37^{\circ} \mathrm{C}$, phenol/chloroform extracted, ethanol precipitated, and resuspended in water at a concentration of $\geqslant 200 \mu \mathrm{g} / \mathrm{ml}$ immediately before sequencing.

In the case of HST2, 26 transposon recombinants were sequenced manually using Sequenase T7 polymerase (U.S. Biochemical) according to the manufacturers instructions. In addition to the transposon-specific primers (SD110 and SD111), the $\mathrm{T} 3$ and $\mathrm{T} 7$ primer sites flanking the insert were also used to recover the terminal sequences. For $H S T 1$ and $H S T 3$, a smaller number of premapped, optimally spaced AT-2 transposon insertions were sequenced with Taq polymerase (Perkin-Elmer) using Prizm kit dye-terminator cycle sequencing in conjunction with an Applied Biosystems 373A Stretch automated sequencer. Sequencing reactions intiated from eight AT-2 insertions (using primers SD118 and SD119) plus the T3 and T7 primers yielded the complete HST1 sequence. Reactions from 12 transposon insertions (plus the T3 and T7 sites) yielded the complete HST3 sequence. Sequences were assembled and analyzed using Sequencher software (Genecodes, Inc., Ann Arbor, MI) for the Macintosh computers.

\section{Sequence and data base analysis}

Multiple sequence alignments were performed using the PILEUP program of the GCG software package (University of Wisconsin, Madison, WI) with preset options, adjusted as described in Figure 1. Homologs of SIR2 from S. aureus (SASIR2, M32103) and $R$. norvegicus (RnSIR2, D37934) were sequenced as flanking DNA of the lac operon (Oskouian and Stewart 1990) and the 5E5 antigen cDNA (Yoshimura et al. 1990) respectively, allowing their identification through TBLASTN data base searches. The SaSIR2 sequence was corrected by insertion of a single $C$ at position 692 based on sequencing of the region cor- 
responding to nucleotides 645-941, amplified by PCR on a $S$. aureus colony. Although the SaSIR 2 homolog sequence is complete, the RnSIR2 sequence probably is not. Because the cDNA sequence encodes the homolog on the minus strand upstream of and slightly overlapping the 5E5 antigen, it is unclear where the RnSIR2 ORF begins. For the alignment we truncated this sequence at its amino terminus, guided by similarities to the human sequence. The partial $H$. sapiens homolog (HsSIR2) sequence was assembled into a contig encoding a single ORF from expressed sequence tag (EST) data base sequences (H14246, R24561, F11858, T66100, R15236, Z42780, T81280, T83544, R25838, R13142, H11816, R56596, F00550, R16487, R07921, $\mathrm{H} 06578$, and H06548) and additional sequencing of T66100 cDNA. The $K$. lactis homolog sequence was reported previously (Chen and Clark-Walker 1994). The C. elegans genome sequencing effort recently identified two tightly linked SIR2 homologs (Z50177). Additional ESTs from A. thaliana and mouse were also found (not shown). Pairwise comparisons were performed using the GAP program of the GCG software package. Data base searches were performed at the National Center for Biotechnology Instruction (NCBI) using the BLAST network service. Some of the homologous EST sequences were identified through the XREF project (Bassett et al. 1995).

\section{Strains, media, and disruption mutations}

Yeast strains are derived from strains YPH499, 500 and 501 (Sikorski and Hieter 1989), FY2 (Winston et al. 1995), or W303 and are described in Table 1. All media used were as described (Rose et al. 1990).

Multiple deletion/disruption mutations were analyzed. The following alleles represent partial deletion/disnuption mutations: hst $1 \Delta 2:: L E U 2$, hst $2 \Delta 1:: T R P 1$, hst $4 \Delta 1:: U R A 3$, hst4D1::TRP1. The following alleles represent complete deletion/disruption mutations (in which the entire HST ORF is replaced): hst1 $\triangle 3:: T R P 1$, hst2 $\triangle 2:: T R P 1$, hst3 $33:: H I S 3$, hst3 $\triangle 3:: T R P 1$ sir2 $\triangle 2:: T R P 1$. In no case tested did the phenotypes of partial or full deletion alleles differ; details will be published elsewhere.

\section{Plasmids}

Plasmids for overexpression studies were constructed in the $A D E 22 \mu$ plasmid pJK204 (Keeney et al. 1995) or in the URA3 $2 \mu$ plasmid YEp352 (Hill et al. 1986). For pLP317, the SIR2 overexpression plasmid, the $2.7-\mathrm{kb}$ Bst NI fragment containing the gene was treated with VENT DNA polymerase (New England BioLabs) and subcloned into YEp352 digested with SmaI. The HST1 overexpression plasmid pLP316 was made by subcloning the 2.2-kb SacI fragment containing HST1 from pGAD42C into SacI-digested YEp352. pCAR143 (used for sequence analysis of HST2) is pCAR135 cut with $\mathrm{Xbal}$ and recircularized. The $H S T 2$ overexpression plasmid pCAR172 was constructed by subcloning the $\mathrm{XbaI}-\mathrm{Cl} a \mathrm{I}$ fragment containing HST2 from pCAR143 into pJK204, which had been digested with ClaI and NheI. For pCAR211, the HST3 overexpression plasmid, the 3.7-kb HindIII fragment from pCAR192 was first subcloned into pRS415 making pCAR202. pCAR202 was digested with SalI, treated with Klenow to create a blunt end, and then digested with $\mathrm{XbaI}$. The resulting $3.7-\mathrm{kb}$ fragment containing HST3 was ligated into pJK204, which had been linearized with ClaI, blunted with Klenow, and then digested with NheI. Plasmid pCAR206 used for the sequence analysis of HST3 was constructed by cloning the $1.9-\mathrm{kb}$ PstI-HpaI fragment containing HST3 from pCAR202 into pMOB (Strathmann et al. 1991) digested with PstI and Ecl136II.
Quantitative mating analysis with high copy HST genes

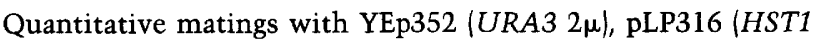
URA3 $2 \mu$ ), pLP317 (SIR2 URA3 $2 \mu$ ) transformants of sir2 strains LPY11 and LPY1393 were performed essentially as described by Sprague (1991). The assays to evaluate $S U P 3_{a m}$ expression by measuring $\operatorname{trp} 1_{a m}$ suppression were performed by diluting saturated overnight cultures of LPY1539, growing in SC-Ura to an $\mathrm{OD}_{600}$ of 1.0 , and making serial fivefold dilutions. Three microliters of each dilution were spotted onto plates to select for plasmid only (SC-Ura plates) and for $\operatorname{trp} 1_{a m}$ suppression in the presence of plasmid (SC-Trp-Ura plates).

\section{Chromosome loss rate determination}

Chromosome loss assays were performed essentially as described, using the chromosome fragments "CEN3 left" and "RAD2 distal" (Spencer et al. 1990). Diploid strains harboring a fragment were taken from plates selective for the fragment, resuspended in $\mathrm{H}_{2} \mathrm{O}$, briefly sonicated, and plated at a density of 200 per plate onto supplemented minimal plates including 6 $\mu \mathrm{g} / \mathrm{ml}$ of adenine (to enhance red pigment formation in ade2 strains). After 5 days at $30^{\circ} \mathrm{C}$, these plates were incubated further at $4^{\circ} \mathrm{C}$ for $>1$ week before scoring. Loss rates were determined by counting the number of red:pink half-sectored colonies (1:0 events) and the number of red:white half-sectored colonies (2:0 events) and dividing by the number of fully pink colonies (containing one chromosome fragment).

\section{Flow cytometry and microscopy}

For flow cytometric analysis, approximately $1 \times 10^{6}$ cells from yeast strains grown to early- to mid-log phase $\left(\mathrm{OD}_{600}=0.3-0.8\right)$ were collected and fixed overnight at $4^{\circ} \mathrm{C}$ in $70 \%$ ethanol, $0.2 \mathrm{M}$ Tris-Cl (pH 7.6). After two washes, cells were resuspended in 0.2 $\mathrm{ml}$ of $1 \mu \mathrm{g} / \mathrm{ml}$ RNase A, $0.2 \mathrm{M}$ Tris- $\mathrm{HCl}(\mathrm{pH} 7.6)$ and incubated at $37^{\circ} \mathrm{C}$ for $4-6 \mathrm{hr}$. Cells were then washed twice, resuspended in $1 \mathrm{ml}$ of $0.2 \mathrm{M}$ Tris- $\mathrm{HCl}(\mathrm{pH} 7.6)$ containing $3 \mu \mathrm{g} / \mathrm{ml}$ of propidium iodide (Sigma), and incubated for $1-5$ days at $4^{\circ} \mathrm{C}$. Flow cytometric analysis was performed on a Coulterepics 752 apparatus with a gain of 10 and high voltage of $935 \mathrm{~V}$. Propidium iodide-stained cells were excited with $400 \mathrm{~mW}$ of $488 \mathrm{~nm}$ light and integrated red $(\geqslant 590 \mathrm{~nm})$ fluorescence was recorded.

For nuclear morphology determination, early- to mid-log phase cells were collected. Nuclei and microtubules were stained and visualized as described (Doheny et al. 1993). Phase contrast microscopy was performed on live or fixed cells.

\section{Viability determination}

Individual log phase cells grown in liquid YPD $\left(\mathrm{OD}_{600}=0.5\right)$ were micromanipulated on YPD plates and grown at $30^{\circ} \mathrm{C}$ and assessed for colony formation. For determination of viability of large-budded cells, only those cells in which the daughter cell was $>50 \%$ the size of the mother cell were micromanipulated.

\section{Acknowledgments}

We thank M. Goebl for pointing out limited homology between HST2 and SIR2, R. Sternglanz for piquing our interest, and J. Rine and P. Hieter for uplifting discussions. We gratefully acknowledge M. Derbyshire and J. Strathern for communication of data regarding their independent discovery of the HST gene family prior to publication. C.B.B. and J.D.B. thank R. Brachmann for support, Toby for patience, D. Bassett Jr. for XREFdb expertise, and J. Fluke for assistance with FACScan analysis. 
J.M.S. and L.P. thank C. Yan and T. Mélèse for their important early contributions. Sequence analysis was supported by a sponsored research agreement with the Perkin-Elmer Corporation, Applied Biosystems Division, to S.E.D. and J.D.B. Supported in part by a National Science Foundation (NSF) Predoctoral Fellowship to C.B.B., an American Cancer Society (ACS) Postdoctoral Fellowship to S.E.D., funding from the Pew Charitable Trust and the NSF to L.P., and an ACS Faculty Research Award and National Institutes of Health (NIH) grant to J.D.B.

The publication costs of this article were defrayed in part by payment of page charges. This article must therefore be hereby marked "advertisement" in accordance with 18 USC section 1734 solely to indicate this fact.

\section{References}

Allshire, R.C., J.-P. Javerzat, N.J. Readhead, and G. Cranston. 1994. Position effect variegation at fission yeast centromeres. Cell 76: 157-169.

Aparicio, O.M. and D.E. Gottschling. 1994. Overcoming telomeric silencing-A trans-activator competes to establish gene expression in a cell cycle-dependent way. Genes \& Dev. 8: 1133-1146.

Aparicio, O.M., B.L. Billington, and D.E. Gottschling. 1991. Modifiers of position effect are shared between telomeric and silent mating-type loci in S. cerevisiae. Cell 66: 1279_ 87.

Bassett, D.E. Jr., M.S. Boguski, F. Spencer, R. Reeves, M. Goebl, and P. Hieter. 1995. Comparative genomics, genome crossreferencing and XREFdb. Trends Genet. 11: 372-373.

Bell, S.P., R. Kobayashi, and B. Stillman. 1993. Yeast origin recognition complex functions in transcription silencing and DNA replication [see comments]. Science 262: 1844-1849.

Braunstein, M., A.B. Rose, S.G. Holmes, C.D. Allis, and J.R. Broach. 1993. Transcriptional silencing in yeast is associated with reduced nucleosome acetylation. Genes \& Dev. 7: 592604.

Buchman, A.R., W.J. Kimmerly, J. Rine, and R.D. Kornberg. 1988. Two DNA-binding factors recognize specific sequences at silencers, upstream activating sequences, autonomously replicating sequences, and telomeres in Saccharomyces cerevisiae. Mol. Cell. Biol. 8: 210-25.

Butner, K. and C.W. Lo. 1986. Modulation of $t k$ expression in mouse pericentromeric heterochromatin. Mol. Cel. Biol. 6: $4440-4449$.

Chen, X.-J. and G.D. Clark-Walker. 1994. sir2 mutants of Kluyveromyces lactis are hypersensitive to DNA-targeting drugs. Mol. Cell. Biol. 14: 4501-4508.

Chien, C.T., P.L. Bartel, R. Sternglanz, and S. Fields. 1991. The two-hybrid system: A method to identify and clone genes for proteins that interact with a protein of interest. Proc. Natl. Acad. Sci. 88: 9578-9582.

Cockell, M., F. Palladino, T. Laroche, G. Kyrion, C. Liu, A.J. Lustig, and S.M. Gasser. 1995. The carboxy termini of Sir4 and Rapl affect Sir3 localization--Evidence for a multicomponent complex requiredfor yeast telomeric silencing. $/$. Cell Biol. 129: 909-924.

Dagberg, B. and B.E. Uhlin. 1992. Regulation of virulence-associated plasmid genes in enteroinvasive Escherichia coli. $J$. Bacteriol. 174: 7606-7612.

Dani, G.M. and V.A. Zakian. 1983. Mitotic and meiotic stability of linear plasmids in yeast. Proc. Natl. Acad. Sci. 80: 34063410 .

Derbyshire, M.K., K.G. Weinstock, and J.N. Strathern. 1995. HST1, a new member of the SIR2 fanily of genes. Yeast (in press).
Devine, S.E. and J.D. Boeke. 1994. Efficient integration of artificial transposons into plasmid targets in vitro: A useful tool for DNA mapping, sequencing and genetic analysis. Nucleic Acids Res. 22: 3765-3772.

Doheny, K.F., P.K. Sorger, A.A. Hyman, S. Tugendreich, F. Spencer, and P. Hieter. 1993. Identification of essential components of the $S$. cerevisiae kinetochore. Cell 73: 761-774.

Fangman, W.L. and B.J. Brewer. 1992. A question of time: Replication origins of eukaryotic chromosomes. Cell 71:363366.

Ferguson, B.M. and W.L. Fangman. 1992. A position effect on the time of replication origin activation in yeast. Cell 68: 333-339.

Foss, M., F.J. McNally, P. Laurenson, and J. Rine. 1993. Origin recognition complex (ORC) in transcriptional silencing and DNA replication in S. cerevisiae. Science 262: 1838-1844.

Gatti, M., D.A. Smith, and B.S. Baker. 1983. A gene controlling condensation of heterochromatin in Drosophila melanogaster. Science 221: 83-85.

Gerring, S.L., F. Spencer, and P. Hieter. 1990. The CHL1 (CTF1) gene product of Saccharomyces cerevisiae is important for chromosome transmission and normal cell cycle progression in G2/M. EMBO I. 9: 4347-4358.

Gottlieb, S. and R.E. Esposito. 1989. A new role for a yeast transcriptional silencer gene, SIR2, in regulation of recombination in ribosomal DNA. Cell 56: 771-776.

Gottschling, D.E. 1992. Telomere-proximal DNA in Saccharomyces cerevisiae is refractory to methyltransferase activity in vivo. Proc. Natl. Acad. Sci. 89: 4062-4065.

Gottschling, D.E., O.M. Aparicio, B.L. Billington, and V.A. Zakian. 1990. Position effect at S. cerevisiae telomeres: Reversible repression of Pol II transcription. Cell 63: 751-762.

Guillen, N., M. Amar, and L. Hirschbein. 1985. Stabilized noncomplementing diploids (Ncd) from fused protoplast products in B. Subtilis. EMBO J. 4: 1333-1338.

Hecht, A., T. Laroche, S. Strahl-Bolsinger, S.M. Gasser, and M. Grunstein. 1995. Histone $\mathrm{H} 3$ and H4 N-termini interact with SIR3 and SIR4 proteins: A molecular model for the formation of heterochromatin in yeast. Cell 80: 583-592.

Hieter, P., C. Mann, M. Snyder, and R.W. Davis. 1985. Mitotic stability of yeast chromosomes: A colony color assay that measures nondisjunction and chromosome loss. Cell 40: 381-392.

Hill, J.E., A.M. Myers, T.J. Koerner, and A. Tzagoloff. 1986. Yeast/E.coli shuttle vectors with multiple unique restriction sites. Yeast 2: 163-167.

Hirano, T., S. Funahashi, T. Uemura, and N. Yanagida. 1986. Isolation and characterization of Schizosaccharomyces cut mutants that block nuclear division but not cutokenesis. EMBO J. 5: 2973-2979.

Johnson, L.M., P.S. Kayne, E.S. Kahn, and M. Grunstein. 1990. Genetic evidence for an interaction between SIR3 and histone H4 in the repression of the silent mating loci in Saccharomyces cerevisiae. Proc. Natl. Acad. Sci. 87: 62866290.

Kayne, P.S., U.J. Kim, M. Han, J.R. Mullen, F. Yoshizaki, and M. Grunstein. 1988. Extremely conserved histone H4 N terminus is dispensable for growth but essential for repressing the silent mating loci in yeast. Cell 55: 27-39.

Keeney, J.B., K.B. Chapman, V. Lauermann, D.F. Voytas, S.U. Åström, U. von Pawel-Rammingen, A. Byström, and J.D. Boeke. 1995. Multiple molecular determinants for retrotransposition in a primer tRNA. Mol. Cell. Biol. 15: 217226.

Kennedy, B.K., N.R. Austriaco Jr., J. Zhang, and L. Guarente. 1995. Mutation in the silencing gene SIR4 can delay aging in 
S. cerevisiae. Cell 80: 485-496.

Laman, H., D. Balderes, and D. Shore. 1995. Disturbance of normal cell cycle progression enhances the establishment of transcriptional silencing in Saccharomyces cerevisiae. Mol. Cell. Biol. 15: 3608-3617.

Laurenson, P. and J. Rine. 1992. Silencers, silencing, and heritable transcriptional states. [Review]. Microbiol. Rev. 56: 543-560.

Lin, R., J.W. Leone, R.G. Cook, and C.D. Allis. 1989. Antibodies specific to acetylated histones document deposition- and transcription-related histone acetylation in Tetrahymena. J. Cell Biol. 108: 1577-1588.

Loo, S. and J. Rine. 1994. Silencers and domains of generalized repression. Science 264: 1768-1771.

. 1995. Silencing and heritable domains of gene expression. Annu. Rev. Cell Biol. Dev. 11: 519-548.

Mahoney, D.J. and J.R. Broach. 1989. The HML mating-type cassette of Saccharomyces cerevisiae is regulated by two separate but functionally equivalent silencers. Mol. Cell. Biol. 9: $4621-4630$.

McCarroll, R.M. and W. Fangman. 1988. Time of replication of yeast centromeres and telomeres. Cell 54: 505-513.

Megee, P.C., B.A. Morgan, B.A. Mittman, and M.M. Smith. 1990. Genetic analysis of histone H4: Essential role of lysines subject to reversible acetylation. Science 247: 841845.

Megee, P.C., B.A. Morgan, and M.M. Smith. 1995. Histone H4 and the maintenance of genome integrity. Genes \& Dev. 9: 1716-1727.

Micklem, G., A. Rowley, J. Harwood, K. Nasmyth, and J.F. Diffley. 1993. Yeast origin recognition complex is involved in DNA replication and transcriptional silencing. Nature 366: 87-89.

Migeon, B. 1994. X-chromosome inactivation: Molecular mechanisms and genetic consequences. Trends Genet. 10: 230235.

Moretti, P., K. Freeman, L. Coodly, and D. Shore. 1994. Evidence that a complex of sir proteins interacts with the silencer and telomere binding protein rapl. Genes \& Dev. 8: 2257-2269.

Nimmo, E.R., G. Cranston and R.C. Allshire. 1994. Telomereassociated chromosome breakage in fission yeast results in variegated expression of adjacent gene. EMBO /. 13: 38013811 .

Oskouian, B. and G.C. Stewart. 1990. Repression and catabolite repression of the lactose operon of Staphylococcus aureus. I. Bacteriol. 172: 3804-3812.

Paetkau, D.W., J.A. Riese, W.S. MacMorran, R.A. Woods, and R.D. Gietz. 1994. Interaction of the yeast RAD7 and SIR3 proteins: Implications for DNA repair and chromatin structure. Genes \& Dev. 8: 2035-2045.

Palladino, F., T. Laroche, E. Gilson, A. Axelrod, L. Pillus and S.M. Gasser. 1993. SIR3 and SIR4 proteins are required for the positioning and integrity of yeast telomeres. Cell 75: 543-555.

Park, E.C. and J.W. Szostak. 1990. Point mutations in the yeast histone $\mathrm{H} 4$ gene prevent silencing of the silent mating type locus HML. Mol. Cell. Biol. 10: 4932-4934.

Pillus, L. and J. Rine. 1989. Epigenetic inheritance of transcriptional states in S. cerevisiae. Cell 59: 637-647.

Renauld, H., O.M. Aparicio, P.D. Zierath, B.L. Billington, S.K. Chhablani, and D.E. Gottschling. 1993. Silent domains are assembled continuously from the telomere and are defined by promoter distance and strength, and by SIR3 dosage. Genes \& Dev. 7: 1133-1145.

Rose, M.D., F. Winston, and P. Hieter. 1990. Methods in yeast genetics. A laboratory course manual. Cold Spring Harbor Laboratory Press, Cold Spring Harbor, New York.

Schaeffer, P., B. Cami, and R.D. Hotchkiss. 1976. Fusion of bacterial protoplasts. Proc. Nat1. Acad. Sci. 73: 2151-2155.

Schnell, R. and J. Rine. 1986. A position effect on the expression of a tRNA gene mediated by the SIR genes in Saccharomyces cerevisiae. Mol. Cell. Biol. 6: 494-501.

Shei, G.J. and J.R. Broach. 1995. Yeast silencers can act as orientation-dependent gene inactivation centers that respond to environmental signals. Mol. Cell. Biol. 15: 3496-3506.

Shero, J.H., M. Koval, F. Spencer, R.E. Palmer, P. Hieter, and D. Koshland. 1991. Analysis of chromosome segregation in Saccharomyces cerevisiae. Methods Enzymol. 194: 749-773.

Shore, D. 1994. RAPl-A protean regulator in yeast. Trends Genet. 10: 408-412.

Sikorski, R.S. and P. Hieter. 1989. A system of shuttle vectors and yeast host strains designed for efficient manipulation of DNA in Saccharomyces cerevisiae. Genetics 122: 19-27.

Singh, J. and A.J. Klar. 1992. Active genes in budding yeast display enhanced in vivo accessibility to foreign DNA methylases: A novel in vivo probe for chromatin structure of yeast. Genes \& Dev. 6: 186-196.

Spencer, F., S.L. Gerring, C. Connelly, and P. Hieter. 1990. Mitotic chromosome transmission fidelity mutants in Saccharomyces cerevisiae. Genetics 124: 237-249.

Sprague, G.F. 1991. Assay of yeast mating reaction. Methods Enzymol. 194: 77-93.

Strathmann, M., B.A. Hamilton, C.A. Mayeda, M.I. Simon, E.M. Meyerowitz, and M.J. Palazzolo. 1991. Transposon-facilitated DNA sequencing. Proc. Natl. Acad. Sci. 88: 12471250.

Sussel, L. and D. Shore. 1991. Separation of transcriptional activation and silencing functions of the RAP1-encoded repressor/activatior protein 1 : Isolation of viable mutants affecting both silencing and telomere length. Proc. Natl. Acad. Sci. 88: 7749-7753.

Sussel, L., D. Vannier, and D. Shore. 1993. Epigenetic switching of transcriptional states: Cis- and trans-acting factors affecting establishment of silencing at the HMR locus in Saccharomyces cerevisiae. Mol. Cell. Biol. 13: 3919-3928.

Terleth, C., C.A. van Sluis, and P. van de Putte. 1989. Differential repair of UV damage in Saccharomyces cerevisiae. $\mathrm{Nu}$ cleic Acids Res. 17: 4433-4439.

Thompson, J.S., X.F. Ling, and M. Grunstein. 1994. Histone h3 amino terminus is required for telomeric and silent mating locus repression in yeast. Nature 369: 245-247.

Weiler, K.S. and B.T. Wakimoto. 1995. Heterochromatin and gene expression in Drosophila. Ann. Rev. Genet. 29: 577605.

Weinert, T.A. and L.H. Hartwell. 1988. The RAD9 gene controls the cell cycle response to DNA damage in Saccharomyces cerevisiae. Science 241: 317-322.

Weinert, T.A., G.L. Kiser, and L.H. Hartwell. 1994. Mitotic checkpoint genes in budding yeast and the dependence of mitosis on DNA replication and repair. Genes \& Dev. 8: 652-665.

Winston, F., C. Dollard, and S.L. Ricupero-Hovasse. 1995. Construction of a set of convenient Saccharomyces cerevisiae strains that are isogenic to S288C. Yeast 11: 53-55.

Wright, J.H., D.E. Gottschling, and V.A. Zakian. 1992. Saccharomyces telomeres assume a non-nucleosomal chromatin structure. Genes \& Dev. 6: 197-210.

Yoshimura, K., K. Akagawa, Y. Nishimura, and K. Uyemura. 1990. A monoclonal antibody 5E5 recognizes an intranuclear antigen selectively present in a subpopulation of the neurons. Brain Res. 509: 161-164. 


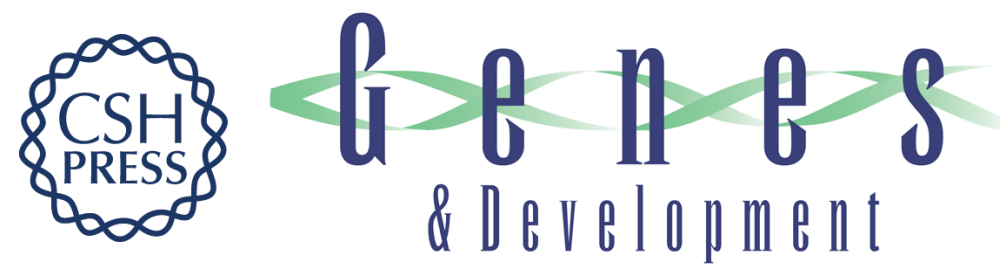

\section{The SIR2 gene family, conserved from bacteria to humans, functions in silencing, cell cycle progression, and chromosome stability.}

C B Brachmann, J M Sherman, S E Devine, et al.

Genes Dev. 1995, 9:

Access the most recent version at doi:10.1101/gad.9.23.2888

References This article cites 71 articles, 38 of which can be accessed free at:

http://genesdev.cshlp.org/content/9/23/2888.full.html\#ref-list-1

License

Email Alerting

Service

Receive free email alerts when new articles cite this article - sign up in the box at the top right corner of the article or click here.

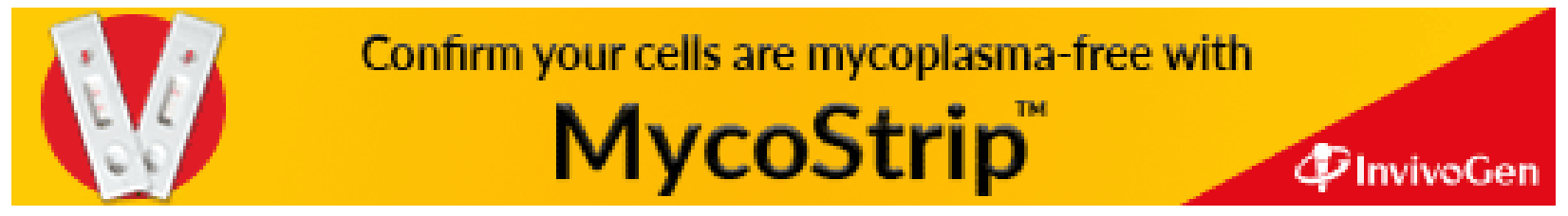

